 \\ A VIBEYOR Robotic Engineering
}

\section{Human-Robot Collaboration}

\section{Hans-Jürgen Buxbaum*}

\section{Laboratory Human Engineering and Robotics, University of Applied Sciences Niederrhein, Germany}

\begin{abstract}
Human-Robot Collaboration (HRC) is gaining more and more importance as a new development direction in robotics. The previously common separating devices are to be eliminated, man and machine are to work in a common process. At present, new fields of application are being opened up in automation, which require both an ergonomically and safety-related suitable design and a philosophical consideration of the resulting socio-technical system.
\end{abstract}

\section{Text}

After four decades of economically and technologically successful use of robots in industrial production, robotics has now reached a new peak: Robots are developed and manufactured with outstanding precision, high positioning accuracy and reliability. Nevertheless, in most industrial applications robots cannot function reliably without humans, as early experiences in the development of robotics have shown. Humans are often needed in the process, for example to correct robot errors.

For some years now, systems of human-robot collaboration (HRC) have been gaining increasing importance. In human-robot collaboration applications, humans and robots work in a common work system without being spatially separated by facilities such as safety fences. Thus a common working space is created, which differs from the previous concepts of separated fenced robot systems. Humans and robots are to carry out work on the same object in the common workspace at the same time. The aim is to maintain the human being with his abilities as an active member of the production and at the same time to increase productivity through automation.

Collaboration is characterized above all by the proximity and form of cooperation between human and robot. On closer inspection, there are even more striking features. For example, robots often have physical characteristics that they share with humans; for example, they are often referred to as robot arm or hand. They also have similar characteristics in terms of interaction and communication, and in some areas of application even natural language skills. In view of the socio-technical system that is obviously emerging, the existence of humans and robots, their bodies, their presence in space, their relationships and similarities - and ethical questions of all kinds - are of philosophical interest.

HRC systems are supposed to support or relieve in monotonous or power-consuming work,

*Corresponding author: Hans-Jürgen Buxbaum, Laboratory Human Engineering and Robotics, University of Applied Sciences Niederrhein, Reinarzstraße 49, 47805 Krefeld, Germany

Accepted: January 25, 2020; Published: January 27, 2020

Copyright: (C) 2020 Hans-Jürgen B. This is an open-access article distributed under the terms of the Creative Commons Attribution License, which permits unrestricted use, distribution, and reproduction in any medium, provided the original author and source are credited.

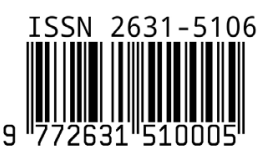

Hans-Jürgen. Int J Robot Eng 2020, 5:021 
as for example in the assembly of threaded screws on geometrically determined components. Today many leading manufacturers of industrial robots develop special HRC robots. Due to collaboration and the elimination of fences, occupational safety and ergonomics are increasingly coming into focus. Intensive research is currently being carried out on workplace design, for example with regard to distraction using eye tracking methods, with regard to safety perception and situation awareness, the predictability of robot movements or task allocation. HRC test benches for full-scope simulation are also under development.

Required for a successful implementation of HRC in industrial applications are lightweight robots with force detection or safety shutdown. In the sense of a versatile production they have to be scalable and flexible in the process. Important is the relief of the workers from monotonous or physically straining work as well as a guaranteed safety by compliance with the machine guidelines and standards. Programming and operation must be simplified; ideally, the machine operator should also be able to carry out programming.

Economically reasonable use of $\mathrm{HRC}$ requires a relevant, calculable benefit. First of all the space utilization of productive facilities, which sooner or later leads to space problems in the industry in case of further economic growth. The omission of separating protective devices makes a space-saving realization possible. The more effective use of productive space leads to increased productivity per unit area. Overlapping work areas allow easier process planning, especially in flexible manufacturing with changing work contents. Increasing the flexibility of the productive facilities is the next point. The term "flexibility" or "flexible manufacturing" is not new. As early as the 1980s, flexible manufacturing systems were used in which the variants of a given product range could be called up via information interfaces. At that time, information became a production factor, and the idea of computer-integrated manufacturing (CIM) was born. The concept of flexibility was scientifically discussed, classifications according to product-related and production-related flexibility became commonplace, and product-related flexibility types (variant flexibility and change flexibility) were distinguished from production-related flexibility types (functional flexibility, volume flexi- bility, expansion flexibility and redundancy). The discussion about flexibility in the context of the $\mathrm{HRC}$ is now being held again. The HRC makes special demands and opens new possibilities of flexibility. Often mentioned is the production-related flexibility of location which describes the mobility of the workplaces. Due to the omission of fences or enclosures, workplaces of the HRC are clearly more flexible in terms of location than usual robot cells. For the first time automation systems are created which can be used at different workplaces and for different tasks, e.g. in case of capacity fluctuations. For the first time an application of robotics becomes economically reasonable in small and medium-sized companies.

Furthermore, ergonomics can be improved by taking into account the strengths and weaknesses of the interaction partners, man and machine. The advantages of the human being in the HRC lie in the fast detection, evaluation and reaction. He has a free mobility and the possibility to compensate tolerances and to detect errors at any time. He uses his sensomotoric abilities like seeing, hearing, feeling etc. for this purpose of his own accord. $\mathrm{He}$ is capable of learning, the handling of objects of varying complexity is unproblematic, and the selection and operation of suitable tools is also intrinsically possible. He is able to question, optimise and innovate processes, he is empathic and can also be used flexibly to a large extent. The advantages of the robot in the HRC are precision and repeatability, and it consistently reaches a level that humans can hardly reach and under production conditions rather not continuously maintain. Thus a higher quality can be achieved by the use of robots. Robots can use heavy objects and tools, and it is also possible to handle dangerous objects. Monotonous, repetitive tasks can be ideally transferred to robots. In addition, a robot is available throughout and can therefore also be used in shift models without any problems.

In order to be able to use the potentials resulting from HRC, an adjustment of the operation by simple, efficient and reliable programming is indispensable. The concept of intuitiveness comes up, in the future it will be necessary that the actions of the robot are transparent and comprehensible for humans. Also an ad-hoc task sharing, which overcomes the limitations of previous sequences in Maba-Maba lists to be defined in the pre-field, 
becomes more and more relevant. In current research projects we are working on the optimization of the operation and programming of collaborative robots and thereby examine the predictive ability and the expectation conformity of robot movements in HRC. The idea is to rely on methods of robotic standard path planning to a large extent in order to guarantee an easy programming and op- eration. At the same time the attention focus and situation awareness of the workers is examined in test person trials.

Besides an ergonomically and safety-related suitable design of the HRC, a philosophical consideration of the resulting socio-technical system is necessary. 